\title{
Phase Transitions of an Oscillator Neural Network with a Standard Hebb Learning Rule
}

\author{
Toru Aonishi \\ Department of Systems and Human Science, Graduate School of Engineering Science, Osaka \\ University, 1-3, Machikaneyama-cho, Toyonaka, Osaka 560, Japan
}

(July 14, 2021)

\begin{abstract}
Studies have been made on the phase transition phenomena of an oscillator network model based on a standard Hebb learning rule like the Hopfield model. The relative phase informations - the in-phase and anti-phase, can be embedded in the network. By self-consistent signal-to-noise analysis (SCSNA), it was found that the storage capacity is given by $\alpha_{c}=0.042$, which is better than that of Cook's model. However, the retrieval quality is worse. In addition, an investigation was made into an acceleration effect caused by asymmetry of the phase dynamics. Finally, it was numerically shown that the storage capacity can be improved by modifying the shape of the coupling function.
\end{abstract}

PACS numbers: 87.10.+e, 05.90.+m, 89.70.+c 


\section{INTRODUCTION}

Two hypotheses are available on the carrier of information in the brain. One, the rate coding hypothesis, states that information is represented by the density of spikes. The other, the temporal coding hypothesis, states that information is represented by the timing of neuronal firings, that is, the synchronization of oscillatory neural activities.

Visual information is divided into a number of features, e.g., color, form, motion, and so on, which are processed in parallel in different areas of the brain. von der Malsburg [1] has emphasized the necessity of temporal coding to bind these features, and have called into question conventional models based on rate coding. It should be noted that some physiological phenomena [2] have supported his discussion. Therefore, this discussion may also increase interest in temporal coding.

One merit of temporal coding is that the information processing (e.g., optimizationminimization of an energy function) achieved by temporal coding neurons (i.e., coincidence detectors) is faster than that achieved by rate coding neurons (i.e., integrators) [3].

Recently, therefore, the oscillator neural network has been attracting the attention of a growing number of researchers.

In this paper, we discuss a class of oscillator neural networks that store two-value memory patterns into synchronously oscillating states. This class of oscillator neural networks is difficult to study analytically, and therefore was not discussed in previous works [6,8]. To overcome the difficulty, we employ a powerful method [12] that can be applied to various networks, and derive the memory capacity. Our model is more biologically relevant than previous models [6], 8], since it is based on a real number synapse which is specified by a standard Hebb learning rule.

In previous analyses [6.8.77 and our analysis, a coupling function of a phase equation was approximately assumed as the sine function for mathematical tractability. In a large population of uniformly coupled oscillators with scattered natural frequencies (which corresponds to ferromagnetic models), the behavior of the system is invariant to the shape of the coupling function, that is, the critical variance of the natural frequency causing a phase transition is only dependent on the lowest frequency components of the coupling function [4]. However, the former analyses do not support our analysis, since properties of the cross-talk noise in our system are different from those of scattered natural frequencies which break the mutual entrainment.

In this parer, in order to verify effects of the shape of the coupling function on the performance of a network, we estimate the memory capacity of more realistic models. In addition, we numerically show that, in the case of an associative memory (on the basis of an

oscillator network), gaps in the coupling function between in-phase and anti-phase improve the performance.

\section{ANALYSIS OF AN OSCILLATOR NETWORK BASED ON A STANDARD HEBB LEARNING RULE}

In general, when the coupling is sufficiently weak, the high-dimensional dynamics of a coupled oscillator system can be reduced to the following phase equation [5], 


$$
\frac{d \phi_{i}}{d t}=-\sum_{j=1}^{N} J_{i j} g\left(\phi_{i}-\phi_{j}\right) ; \quad i=1, \cdots, N,
$$

where $N$ is the total number of oscillators, $\phi_{i}$ is the phase of the $i$ th oscillator and $J_{i j}$ denotes a synaptic weight. $g(\phi)$ is a periodic function. Figures 1 (a)(b) show examples of $g(\phi)$ values (sine-like-curves) obtained by the numerical calculation of weakly-coupled BVP (Bonhoeffer-Van der Pol) oscillators.

For mathematical tractability, we approximately assume $g(\phi)=\sin (\phi)$. Then, Eq. 1 is expressed as,

$$
\frac{d \phi_{i}}{d t}=-\sum_{j=1}^{N} J_{i j} \sin \left(\phi_{i}-\phi_{j}\right)
$$

In Cook's model (a Q-state clock model with $\mathrm{Q} \rightarrow \infty$ ) [6], $J_{i j}$ is a complex number synapse specified by a generalized Hebb learning rule [7,8], which can not be easily achieved in biological implementation. Cook's model can be regarded as an extension of Hopfield networks to "multi-states". Here, $J_{i j}$ is specified by the following learning rule,

$$
J_{i j}=\frac{1}{N} \sum_{\mu=1}^{p} \xi_{i}^{\mu} \xi_{j}^{\mu}, \quad \xi_{i}^{\mu}=\exp \left(i \theta_{i}^{\mu}\right)
$$

where $\left\{\theta_{i}^{\mu}\right\}_{i=1, \cdots, N, \mu=1, \cdots, p}$ are phase patterns to be stored in the network, and are randomly assigned to 0 or $\pi$ with a probability of $1 / 2$.

We define a parameter $\alpha$ (loading rate) such that $\alpha=p / N$. This learning rule is equivalent to a standard Hebb learning rule like in the Hopfield model [9], which can be easily achieved in biological implementation: $J_{i j}$ increases between simultaneously firing cells; otherwise, $J_{i j}$ decreases.

In area CA1 of the hippocampus, stimulation on the in-phase of the hippocampal theta rhythm includes a long-term potentiation that can be depontentiated by stimulation on the anti-phase of the theta rhythm [10]. Thus, oscillator network models with the standard Hebb learning are simple and biologically plausible, yet have to be analyzed mathematically 8.11. 11 .

The system (2) has the following potential $V$ :

$$
V=-\frac{1}{2 N} \sum_{i j}^{N} \sum_{\mu}^{p} \cos \left(\left(\phi_{i}-\theta_{i}^{\mu}\right)-\left(\phi_{j}-\theta_{j}^{\mu}\right)\right),
$$

This potential (4) is invariant under the transformation $\phi_{i} \rightarrow \phi_{i}+\beta$ with any $\beta \in \mathbf{R}$. In other words, the equilibrium solution is irresistant to a uniform shift of $\phi_{i}$ (the neutral stability). Hence, the relative phase informations, i.e., the in-phase and anti-phase, can be embedded in the network.

Here, we study the equilibrium properties of system (2) when $p, N \rightarrow \infty$ with fixed $\alpha=p / N$, by using self-consistent signal-to-noise analysis (SCSNA) 12 which is based on the $\mathrm{S} / \mathrm{N}$ analysis to explain the equilibrium properties. The results of applying SCSNA to the Hopfield model [12] and Cook's model [8] coincide with those of the replica theory [13,6]. SCSNA is a powerful method, since we can easily study the equilibrium properties 
of analog-neuron networks without considering the potential, for example, analogue neural networks with asymmetric connections [12].

Defining the order parameters $m_{c}^{\mu}$ and $m_{s}^{\mu}$ (overlap) in a large $N$ limit as,

$$
m_{c}^{\mu}=\frac{1}{N} \sum_{i} \xi_{i}^{\mu} \cos \phi_{i}, \quad m_{s}^{\mu}=\frac{1}{N} \sum_{i} \xi_{i}^{\mu} \sin \phi_{i}
$$

we obtain the following equilibrium condition by setting $d \phi_{i} / d t=0$ in Eq. 2,

$$
\begin{aligned}
\cos \phi_{i} & =X\left(h_{1}^{i}, h_{2}^{i}\right)=h_{1}^{i} / \sqrt{{h_{1}^{i}}^{2}+h_{2}^{i^{2}}}, \\
\sin \phi_{i} & =Y\left(h_{1}^{i}, h_{2}^{i}\right)=h_{2}^{i} / \sqrt{{h_{1}^{i}}^{2}+h_{2}^{i^{2}}}, \\
h_{1}^{i} & =\sum_{\mu}^{p} \xi_{i}^{\mu} m_{c}^{\mu}, \quad h_{2}^{i}=\sum_{\mu}^{p} \xi_{i}^{\mu} m_{s}^{\mu} .
\end{aligned}
$$

Note that we have ignored another solution $\cos \phi_{i}=-X, \sin \phi_{i}=-Y$ by applying the so-called Maxwell rule [11] of statistical mechanics to find a relevant solution. Assuming $m_{c}^{1}, m_{s}^{1}=O(1)$ and $m_{c}^{\mu}, m_{s}^{\mu}=O(1 / \sqrt{N})$ for $\mu>1$, that is, $\xi_{i}^{1}$ as a condensed pattern, and expanding a polynomial around $m_{c}^{\mu}, m_{s}^{\mu}=0(\mu>1)$ to split the cross-talk noise into an effective self-coupling and a Gaussian random variable [12,[1], we obtain the following nine dimensional equations for the order parameters,

$$
\begin{aligned}
m_{c} & =\left\langle\left\langle X\left(x_{1}, x_{2}\right)\right\rangle\right\rangle, \\
m_{s} & =\left\langle\left\langle Y\left(x_{1}, x_{2}\right)\right\rangle\right\rangle, \\
q_{c} & =\left\langle\left\langle X^{2}\left(x_{1}, x_{2}\right)\right\rangle\right\rangle, \\
q_{s} & =\left\langle\left\langle Y^{2}\left(x_{1}, x_{2}\right)\right\rangle\right\rangle, \\
q_{s c} & =\left\langle\left\langle X\left(x_{1}, x_{2}\right) Y\left(x_{1}, x_{2}\right)\right\rangle\right\rangle, \\
C_{1}= & \frac{1-\Lambda}{\sqrt{\alpha}}\left\langle\left\langle\left(\bar{Q}_{1} x_{1}+\bar{Q}_{3} x_{2}\right) X\left(x_{1}, x_{2}\right)\right\rangle\right\rangle, \\
C_{2}= & \frac{1-\Lambda}{\sqrt{\alpha}}\left\langle\left\langle\left(\bar{Q}_{3} x_{1}+\bar{Q}_{2} x_{2}\right) X\left(x_{1}, x_{2}\right)\right\rangle\right\rangle, \\
S_{1}= & \frac{1-\Lambda}{\sqrt{\alpha}}\left\langle\left\langle\left(\bar{Q}_{1} x_{1}+\bar{Q}_{3} x_{2}\right) Y\left(x_{1}, x_{2}\right)\right\rangle\right\rangle, \\
S_{2}= & \frac{1-\Lambda}{\sqrt{\alpha}}\left\langle\left\langle\left(\bar{Q}_{3} x_{1}+\bar{Q}_{2} x_{2}\right) Y\left(x_{1}, x_{2}\right)\right\rangle\right\rangle,
\end{aligned}
$$

where $\langle\langle\cdots\rangle\rangle$ is taken to mean the Gaussian average over $x_{1}, x_{2}$, that is, $\langle\langle\cdots\rangle\rangle=$ $\iint D x_{1} D x_{2} \cdots$, and the above equations satisfy $C_{2}=S_{1}$ and $q_{c}+q_{s}=1$. Note that gauge transformations were performed on variables of the condensed pattern. The pattern superscripts 1 of $m_{c}, m_{s}$ are omitted for brevity. $\Lambda$ and the Gaussian measure $D x_{1} D x_{2}$ are expressed as follows,

$$
\begin{aligned}
\Lambda & =C_{1}+S_{2}+C_{2} S_{1}-C_{1} S_{2} \\
D x_{1} D x_{2} & =\frac{d x_{1} d x_{2}}{2 \pi \sqrt{\operatorname{det} Q}} \exp \left(-\frac{1}{2}\left[x_{1}, x_{2}\right] Q^{-1}\left[\begin{array}{l}
x_{1} \\
x_{2}
\end{array}\right]\right),
\end{aligned}
$$




$$
\begin{aligned}
Q & =\left[\begin{array}{ll}
Q_{1} & Q_{3} \\
Q_{3} & Q_{2}
\end{array}\right] \\
Q^{-1} & =\frac{1}{\operatorname{det} Q}\left[\begin{array}{cc}
Q_{2} & -Q_{3} \\
-Q_{3} & Q_{1}
\end{array}\right]=\left[\begin{array}{cc}
\bar{Q}_{1} & \bar{Q}_{3} \\
\bar{Q}_{3} & \bar{Q}_{2}
\end{array}\right] \\
Q_{1} & =\left(1-S_{2}\right)^{2} q_{c}+2 C_{2}\left(1-S_{2}\right) q_{s c}+C_{2}^{2} q_{s} \\
Q_{2} & =S_{1}^{2} q_{c}+2 S_{1}\left(1-C_{1}\right) q_{s c}+\left(1-C_{1}\right)^{2} q_{s}, \\
Q_{3} & =S_{1}\left(1-S_{2}\right) q_{c}+\left(1-S_{2}-C_{1}+S_{1} C_{2}+S_{2} C_{1}\right) q_{s c} \\
& +C_{2}\left(1-C_{1}\right) q_{s} .
\end{aligned}
$$

$\cos \phi=X\left(x_{1}, x_{2}\right)$ and $\sin \phi=Y\left(x_{1}, x_{2}\right)$ at equilibrium satisfy the following condition,

$$
\begin{gathered}
-\left((1-\Lambda) m_{c}+\sqrt{\alpha} x_{1}\right) \sin \phi+\left((1-\Lambda) m_{s}+\sqrt{\alpha} x_{2}\right) \cos \phi \\
=\alpha\left[C_{2} \sin ^{2} \phi-S_{1} \cos ^{2} \phi+\left(C_{1}-S_{2}\right) \sin \phi \cos \phi\right] .
\end{gathered}
$$

In general, Eq. 17 admits four solutions owing to the effective self-coupling terms corresponding to the right-hand side of Eq. 17. Figure 2 shows intersection points of the left-hand side and the right-hand side of Eq. 17, where the filled circles and open circles correspond to stable solutions and unstable solutions, respectively. Here, we find an available solution by applying the Maxwell rule [11] in thermodynamics. As shown in Fig. 2, there are two enclosed areas $A_{1}$ and $A_{2}$, provided that the left-hand side of Eq. 17 is to be the upper boundary and the right-hand side of Eq. 17 is to be the lower one. According to the Maxwell rule [11], we must select a stable solution with the larger enclosed area.

The above order parameters are analogous with those of the Hopfield model [13]. $q_{c}$, $q_{s}$, and $q_{s c}$ correspond to the so-called Edwards-Anderson order parameter, which measures the local ordering of XY-spins. $C_{1}, C_{2}, S_{1}$, and $S_{2}$ correspond to the susceptibility, which measures the sensitivity to external fields.

The above equations for the order parameters are more complicated than those of Cook's model (three-dimensional equations) [6]. Because patterns stored in Cook's model are uniformly distributed in $[0,2 \pi]$, 2-dimensional Gaussian noise-Eq. 11 is isotropic, which enables us to reduce to three-dimensional equations in terms of the polar coordinate system. However, in the case of our model, 2-dimensional Gaussian noise-Eq. 11 is anisotropic, since phase patterns stored in our model are only assigned to 0 or $\pi$. Thus, we can not reduce to simpler equations any more.

Here, we define a new order parameter $m=\sqrt{m_{c}^{2}+m_{s}^{2}}$ for convenience. The solid curve in Fig. 3 (a) shows the values of $m$ for various values of loading rate $\alpha$, which were obtained by solving the above equations numerically. The plots in Fig. 3(a) show results obtained by numerical simulation with $N=2000$. Note that the solution of the above equations forms an isotropic manifold as shown in Fig. 3(b), because system (2) is invariant to a uniform shift of $\phi_{i}$. Fig. 3(a) shows a cross-section of this manifold.

The theory is in good agreement with the results of this simulation. The storage capacity is given by $\alpha_{c}=0.042$ and at this point the overlaps are $m=0.69$. The storage capacity of our model is about $1 / 3$ as large as that of the Hopfield model. In the case of Cook's model, $\alpha_{c}=0.038$ and at this point, $m=0.899$. Thus, the storage capacity of our model is better than that of Cook's model, but the retrieval quality is worse. 
The dashed line in Fig. 3(a) denotes an unstable solution of the equations for the order parameters; $m_{c}=1, m_{s}=0, q_{c}=1, q_{s}=0, q_{s c}=0, C_{1}=0, C_{2}=0, S_{1}=0$, and $S_{2}=1$. The solution corresponds to the equilibrium solution $\phi_{i}=\theta_{i}{ }^{1}$ (perfect memory states) from Eq. Q. With a large $N$ limit, the equilibrium solution $\phi_{i}=\theta_{i}{ }^{1}$ is unstable.

Let us examine the stability of this solution at a finite $N$. Linearizing equation (2) around $\phi_{i}=\theta_{i}^{1}$, we obtain the following matrix $A$ :

$$
A=\left[\begin{array}{cccc}
J_{11}-\sum_{j}^{N} J_{1 j} \xi_{1}^{1} \xi_{j}^{1} & J_{12} \xi_{1}^{1} \xi_{2}^{1} & \cdots & J_{1 N} \xi_{1}^{1} \xi_{N}^{1} \\
J_{21} \xi_{2}^{1} \xi_{1}^{1} & J_{22}-\sum_{j}^{N} J_{2 j} \xi_{2}^{1} \xi_{j}^{1} & \cdots & J_{2 N} \xi_{2}^{1} \xi_{N}^{1} \\
\vdots & & & \vdots \\
J_{N 1} \xi_{N}^{1} \xi_{1}^{1} & J_{N 2} \xi_{N}^{1} \xi_{2}^{1} & \cdots & J_{N N}-\sum_{j}^{N} J_{N j} \xi_{N}^{1} \xi_{j}^{1}
\end{array}\right],
$$

where we obtain eigenvalue 0 with eigenvector $\vec{r}=[1, \cdots, 1]^{T}$ which corresponds to a uniform shift of $\phi_{i}$, as previously discussed. We numerically calculated the maximum eigenvalue of $A$ for various values of loading rate $\alpha$. Figure $⿴$ indicates the results, where (a) $N=10$, (b) $N=100$, (c) $N=1000$ and (d) $N=2000$. According to Fig. $⿴$, at $p \leq 2$, the system is neutrally stable around $\phi_{i}=\theta_{i}^{1}$, and at $p>2$, the system is unstable, independently of the values of $N$. Thus, we can estimate that the capacity for perfect memory retrieval is given by $p_{c}=2$.

\section{ACCELERATION EFFECT}

As shown in Figs. [1(a)(c), $g(\phi)$ obtained from real systems is asymmetric with respect to the origin, that is, $g(\phi) \neq-g(-\phi)$. In this case, all phase values $\phi_{i}$ continue rotating with a uniform frequency, while keeping the relative states - in-phase and anti-phase. This phenomenon corresponds to the so-called acceleration effect [14]. Let us consider the following perturbed system for Eq. 2,

$$
\frac{d \phi_{i}}{d t}=-\sum_{j=1}^{N} J_{i j}\left(\sin \left(\phi_{i}-\phi_{j}+\sigma\right)-\sin \sigma\right) .
$$

When $\sigma$ is sufficiently small, we obtain the following equation from Eq. (19),

$$
\frac{d \phi_{i}}{d t}=-\sum_{j=1}^{N} J_{i j} \sin \left(\phi_{i}-\phi_{j}\right)-\sigma \sum_{j=1}^{N} J_{i j}\left(\cos \left(\phi_{i}-\phi_{j}\right)-1\right),
$$

where the second term on the right-hand side is a structural perturbation-asymmetry of $g(\phi)$. The neutral stability mode (a uniform shift of $\phi_{i}$ ) of Eq. 2 is broken by this structural perturbation. Solutions in the neighborhood of an equilibrium solution for an unperturbed system can be represented as,

$$
\phi_{i}(t)=\psi_{i}+\omega \tau+\sigma u_{i}(\tau),
$$

where $\tau=\sigma t$ (slow time variable), and $\psi_{i}$ denotes an equilibrium solution for the unperturbed system. $\omega$ represents a frequency of rotation caused by a structural perturbation. $\sigma u_{i}(\tau)$ is a higher-order fluctuation caused by the effects of a perturbation. Substituting Eq. 
21 into Eq. 20, expanding a polynomial around $\sigma=0$ and neglecting higher-order terms, we obtain

$$
\begin{gathered}
\omega[1, \cdots, 1]^{T}=G\left[u_{1}, \cdots, u_{N}\right]^{T} \\
-\left[\sum_{j=1}^{N} J_{1 j}\left(c_{1 j}-1\right), \cdots, \sum_{j=1}^{N} J_{N j}\left(c_{N j}-1\right)\right]^{T}, \\
G=\left[\begin{array}{cccc}
J_{11}-\sum_{j}^{N} J_{1 j} c_{1 j} & J_{12} c_{12} & \cdots & J_{1 N} c_{1 N} \\
J_{21} c_{21} & J_{22}-\sum_{j}^{N} J_{2 j} c_{2 j} & \cdots & J_{2 N} c_{2 N} \\
\vdots & & & \vdots \\
J_{N 1} c_{N 1} & J_{N 2} c_{N 2} & \cdots & J_{N N}-\sum_{j}^{N} J_{N j} c_{N j}
\end{array}\right], \\
c_{i j}=\cos \left(\psi_{i}-\psi_{j}\right),
\end{gathered}
$$

where we obtain $\vec{r}=[1, \cdots, 1]^{T} \in \operatorname{ker} G$ which corresponds to a uniform shift of $\phi_{i}$. We can expect $\operatorname{ker} G=\operatorname{span}\{\vec{r}\}$, since, if other modes with eigenvalue 0 were to exist, the relative states would be broken by a perturbation. We can erase fluctuation term $G\left[u_{1}, \cdots, u_{N}\right]^{T}$ in Eq. 22 taking an inner product between $\vec{r}$ and Eq. 22. Thus, $\omega$ can be expressed as,

$$
\omega=\frac{1}{N} \sum_{i j}^{N} J_{i j}-\frac{1}{N} \sum_{i j}^{N} \sum_{\mu}^{p} \cos \left(\left(\psi_{i}-\theta_{i}^{\mu}\right)-\left(\psi_{j}-\theta_{j}^{\mu}\right)\right),
$$

where the second term on the right-hand side is the potential $V$ of the unperturbed systemEq. 2. Therefore, when $\omega \neq 0$, a stationary solution is transformed into a rotating solution by a structural perturbation - asymmetry of $g(\phi)$.

\section{MEMORY CAPACITY OF REALISTIC MODELS}

In order to verify effects of the shape of the coupling function on the memory capacity, we estimated the memory capacity of weakly-coupled BVP oscillators with diffusional couplings;

$$
\left\{\begin{array}{c}
\tau \dot{x}_{i}=c\left(x_{i}-x_{i}{ }^{2} / 3+y_{i}\right)+\varepsilon \sum_{j} J_{i j}\left(x_{j}-x_{i}\right) \\
\tau \dot{y}_{i}=-\left(x_{i}+b y_{i}-a\right) / c+\varepsilon \sum_{j} J_{i j}\left(y_{j}-y_{i}\right)
\end{array},\right.
$$

When $\varepsilon$ is sufficiently small, Eq. 26 can be reduced to the phase-variable description (1). Figures 1(a)(b) show $g(\phi)$ values obtained by the numerical calculation, where (a) $a=0$, $b=-0.5, c=5.0$, and (b) $a=0, b=0, c=5.0$. We started the numerical calculation using phase equation (1) with raw $g(\phi)$ values, where $\mathrm{N}=1000$.

As previously discussed, by the asymmetry of $g(\phi)$, all phase values $\phi_{i}$ went on rotating with a uniform frequency, while keeping the relative states - in-phase and anti-phase. By using the pattern overlap $m=\sqrt{m_{c}^{2}+m_{s}^{2}}$, we can observe the macroscopic relative states, since $\left(\sum_{i} \xi_{i} \cos \left(\omega \tau+\phi_{i}\right)\right)^{2}+\left(\sum_{i} \xi_{i} \sin \left(\omega \tau+\phi_{i}\right)\right)^{2}=\left(\sum_{i} \xi_{i} \cos \phi_{i}\right)^{2}+\left(\sum_{i} \xi_{i} \sin \phi_{i}\right)^{2}$.

Figures 5(a)(b) show final values of $m$ versus $\alpha$. The plots were obtained by numerical simulation. The solid curves were obtained by $\operatorname{SCSNA}(g(\phi)=\sin (\phi))$. In Fig. 5(a), the theory is consistent with the results from the simulation. In Fig. 5(b), the memory capacity 
is larger than the approximated system (Eq. 2). These simulations showed that the gaps of $g(\phi)$ in Fig. 1(b) improved the performance of the network.

BVP oscillators consist of an activator $x_{i}$ and an inhibitor $y_{i}$. The movement of the activator is rapid like a pulse wave, but that of the inhibitor is gentle like a sine wave. The gaps in $g(\phi)$ in Fig. [1(b) were caused by a rapid phase-locking of the activator.

We numerically estimated the memory capacity of the artificial model with the following $g(\phi)$ values,

$$
\begin{gathered}
g(\phi)=\left\{\begin{array}{cc}
\sin (\phi), & -\frac{\pi}{2}+2 k \pi<\phi<\frac{\pi}{2}+2 k \pi \\
\gamma \sin (\phi), & \text { otherwise }
\end{array}\right. \\
k=\cdots,-3,-2,-1,0,1,2,3, \cdots,
\end{gathered}
$$

where $\gamma$ is the control parameters for the length of the gaps. Figures $6(a)(b)(c)(d)$ show final values of $m$ and the boundary of attraction versus $\alpha$, where (a) $\gamma=1.0$, (b) $\gamma=0.5$, $\gamma=0.25$, and (c) $\gamma=0.05$. In Fig. 6(c), the memory capacity is larger than that of the original system $(\gamma=1.0)$, and the width of the basin is almost the same as that in Fig. 6(a). In Fig. E(d), both the memory capacity and the width of the basin are worse than those in Fig. 6. (c). From these figures, we could guess that the optimal gap is given by $\gamma \sim 0.25$.

\section{CONCLUSION}

Studies were made on the phase transitions of an oscillator neural network model based on a standard Hebb learning rule like in the Hopfield model. By SCSNA, it was found that the storage capacity is given by $\alpha_{c}=0.042$. In addition, it was numerically shown that the gaps in the coupling function between in-phase and anti-phase improve the performance. This phenomenon show that a realistic model, i.e., the pulse-coupled oscillator has a good performance compared to a simple "XY-spin".

The storage capacity of the Hopfield model can be improved by replacing the usual monotonic output function with a nonmonotonic one [15]. The susceptibility becomes negative by the nonmonotonicity of the output function, so the variance of the cross-talk noise decreases. However, under the present conditions, we can not determine whether the properties of the gaps of the coupling function might be analogous to those of the nonmonotonicity in the Hopfield model. In future work, we need to expand our theory to allow it to deal with general $g(\phi)$ values.

In Cook's model and our model, only replica-symmetric solutions are considered. SCSNA is based on replica-symmetry, because the Gaussian ansatz for the cross-talk noise in SCSNA corresponds to the replica-symmetry ansatz in the replica theory. It is well-known that replica-symmetry breaking (RSB) [16] occurs in Cook's model, since the entropy of this system becomes a negative value [17]. We can also guess that replica-symmetry breaking occurs in our model. Therefore, we need to derive RSB solutions [18] of the oscillator network model as in the Hopfield model. However, the derivation of the RSB solutions is more difficult than with the Hopfield model.

The author thanks Dr. K. Kurata and Dr. M. Okada for their valuable discussions. This work was partially supported by Grants-in-Aid for the Encouragement of Young Scientists No. 0782 and JSPS Research Fellowships for Young Scientists. 


\section{REFERENCES}

[1] For example, C. von der Malsburg, Am I thinking assemblies?, in Brain Theory (Springer-Verlag, 1984).

[2] For example, A. K. Engel, P. König, A. K. Kreiter, T. B. Schillen, and W. Singer, Trends in Neuroscience, 15[6], 218 (1992).

[3] J. J. Hopfield and A. V. M. Hertz, Proc. Natl. Acad. Sci. USA, 92, 6655 (1995).

[4] H. Daido, Pro. of Theo. Phys., 89[4], 929 (1993).

[5] G. B. Ermentrout, Journal of Mathematical Biology, 6, 327 (1981); Y. Kuramoto, Chemical oscillations, waves and turbulence (Springer-Verlag, 1984).

[6] J. Cook, J. Phys. A: Math. Gen., 22, 2057 (1989).

[7] T. Aoyagi and K. Kitano, Physical Review E, 55, 7424 (1997).

[8] K. Okuda, (unpublished).

[9] J. J. Hopfield, Proc. Natl. Acad. Sci. USA, 79, 2554 (1982).

[10] C. Hölscher, R. Anwyl, and M. J. Rowan, J. Neuroscience, 17[16], 6470 (1997).

[11] T. Fukai and M. Shiino, Europhys. Lett., 26[9], 647 (1994); T. Fukai and M. Shiino, Neural Computation, 7, 529 (1995).

[12] M. Shiino and T. Fukai, J. Phys. A: Math. Gen., 25, L375 (1992).

[13] D. J. Amit, H. Gutfreund, and H. Sompolinsky, Physical Review Letters, 55[14], 1530 (1985).

[14] C. Meunier, Biol. Cybern., 67, 155 (1992); S. Mizuno and K. Kurata, Technical report of IEICE (in Japanese), NC94-15, 9 (1994).

[15] M. Morita, Neural Networks, 6, 115 (1993).

[16] J. R. L. de Almeida, and D. J. Thouless, J. Phys. A: Math. Gen., 11[5], 983 (1978).

[17] T. Aoyagi, Private communication, (1996).

[18] G. Parisi, J. Phys. A: Math. Gen., 13, L115 (1980). 


\section{FIGURES}

FIG. 1. (a)(b) Examples of $g(\phi)$ which are obtained by the numerical calculation of weakly-coupled BVP oscillators, i.e., Eq. 26, where (a) $a=0, b=-0.5, c=5.0$, and (b) $a=0, b=0, c=5.0$.

FIG. 2. A schematic diagram of the Maxwell rule to select relevant solutions to the equilibrium condition.

FIG. 3. (a) Values of $m$ versus loading rate $\alpha$. The solid curve denotes a stable equilibrium solution, and the dashed line shows an unstable equilibrium solution which corresponds to $\phi_{i}=\theta_{i}^{1}+\beta$ with any $\beta \in \mathbf{R}$. These lines are obtained by SCSNA. The plots show results obtained by numerical simulation with $N=2000$. (b) Manifold of the solution. Figure 3(a) shows a cross-section of this manifold.

FIG. 4. The maximum eigenvalue of matrix $A$ versus the loading rate $\alpha$, where (a) $N=10$, (b) $N=100$, (c) $N=1000$ and (d) $N=2000$. At $p \leq 2$, the system is neutrally stable around $\phi_{i}=\theta_{i}^{1}$, and at $p>2$, the system is unstable, independently of the values of $N$.

FIG. 5. (a)(b) Values of $m$ versus $\alpha$. The plots are obtained by numerical calculation using phase equation (1) with the $g(\phi)$ values shown in Figs. 1(a)(b), respectively; $\mathrm{N}=1000$. The solid curves are obtained by SCSNA $(g(\phi)=\sin (\phi))$.

FIG. 6. Basin of attraction and memory capacity of an artificial modal with controllable gaps. The upper data points represent the equilibrium overlap. The lower data points denote the boundary of attraction. All of the data is obtained by numerical simulation when $N=512$ for five trials, except Fig. 6(a) $(\mathrm{N}=1024)$. The error bars indicate standard deviations. (a) $\gamma=1.0$. (b) $\gamma=0.5$. (c) $\gamma=0.25$. (d) $\gamma=0.05$. 

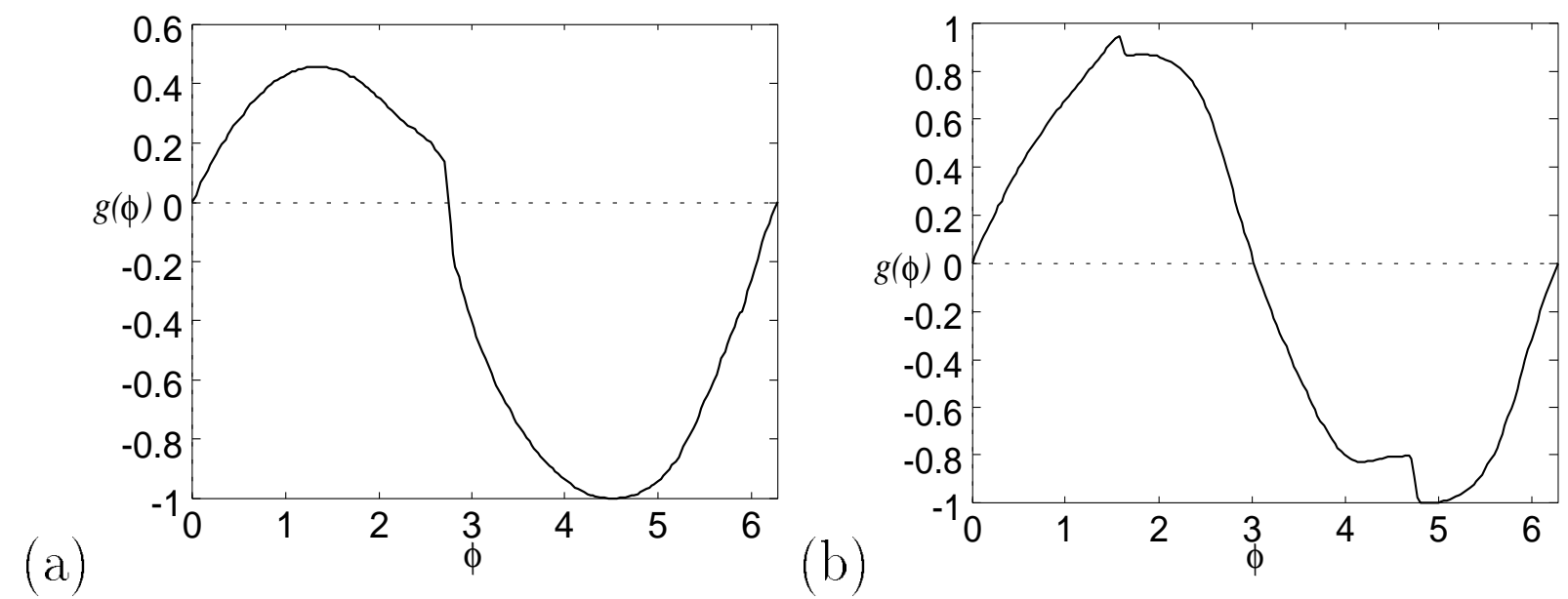

Figure 1

T. Aonishi 


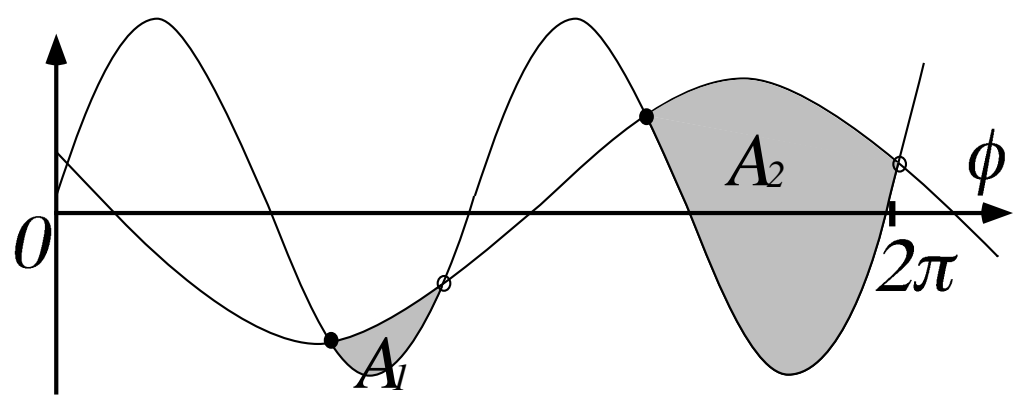

Figure 2

T. Aonishi 

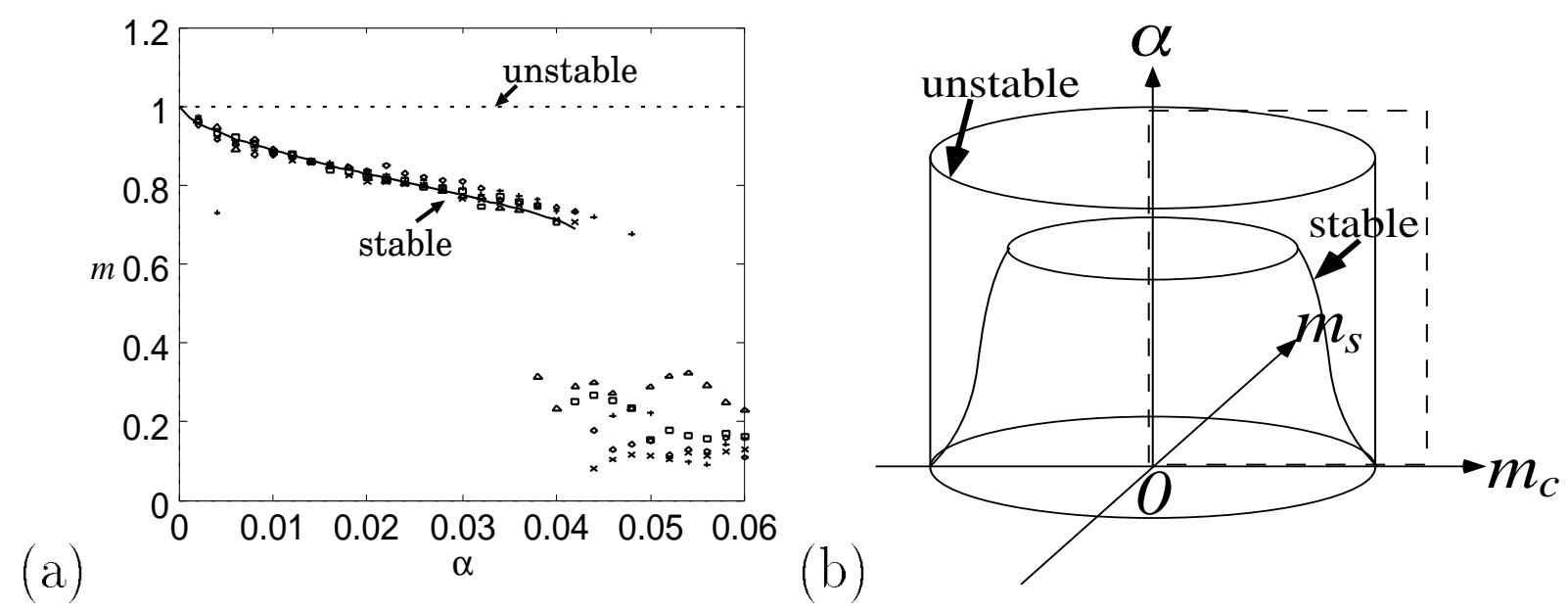

Figure 3

T. Aonishi 

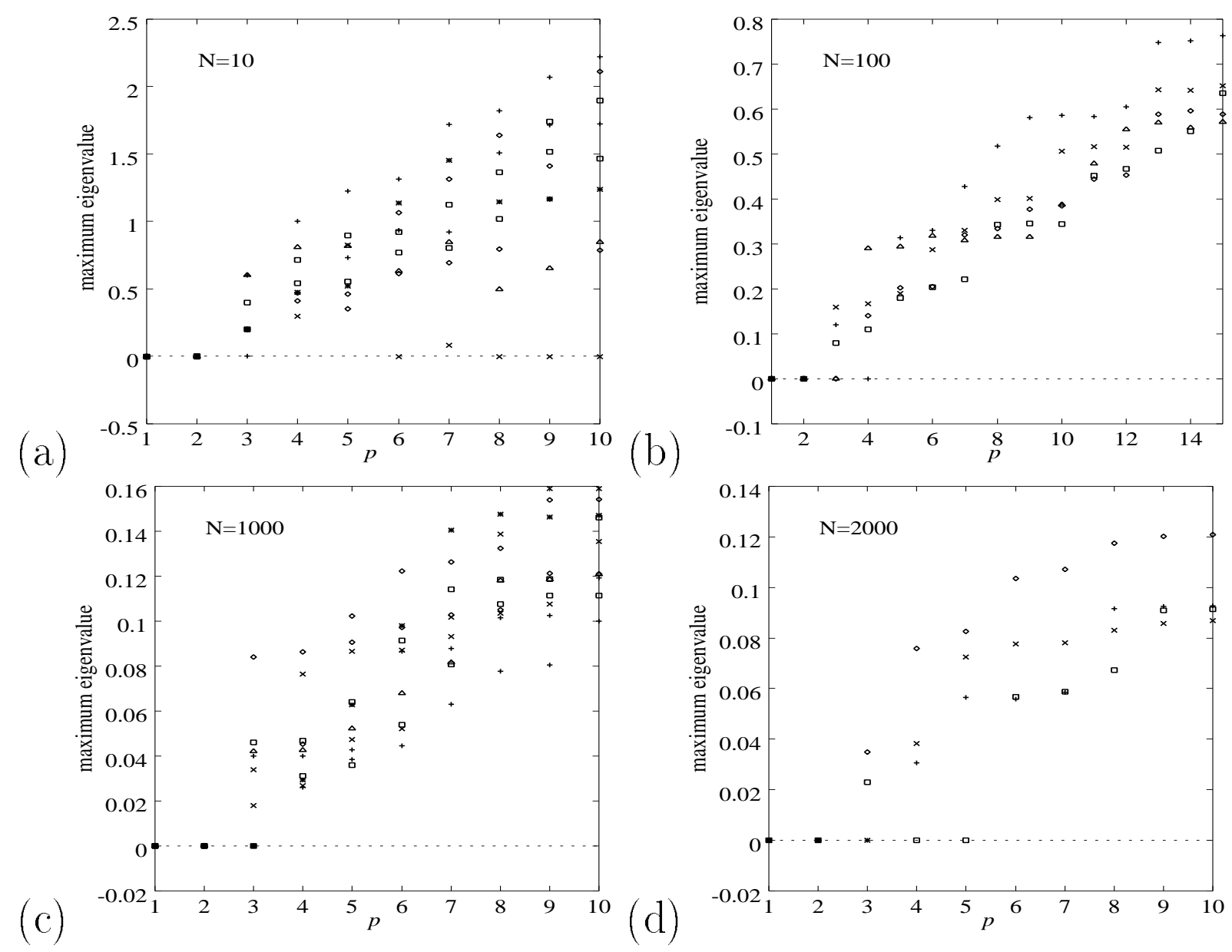

Figure 4

T. Aonishi 

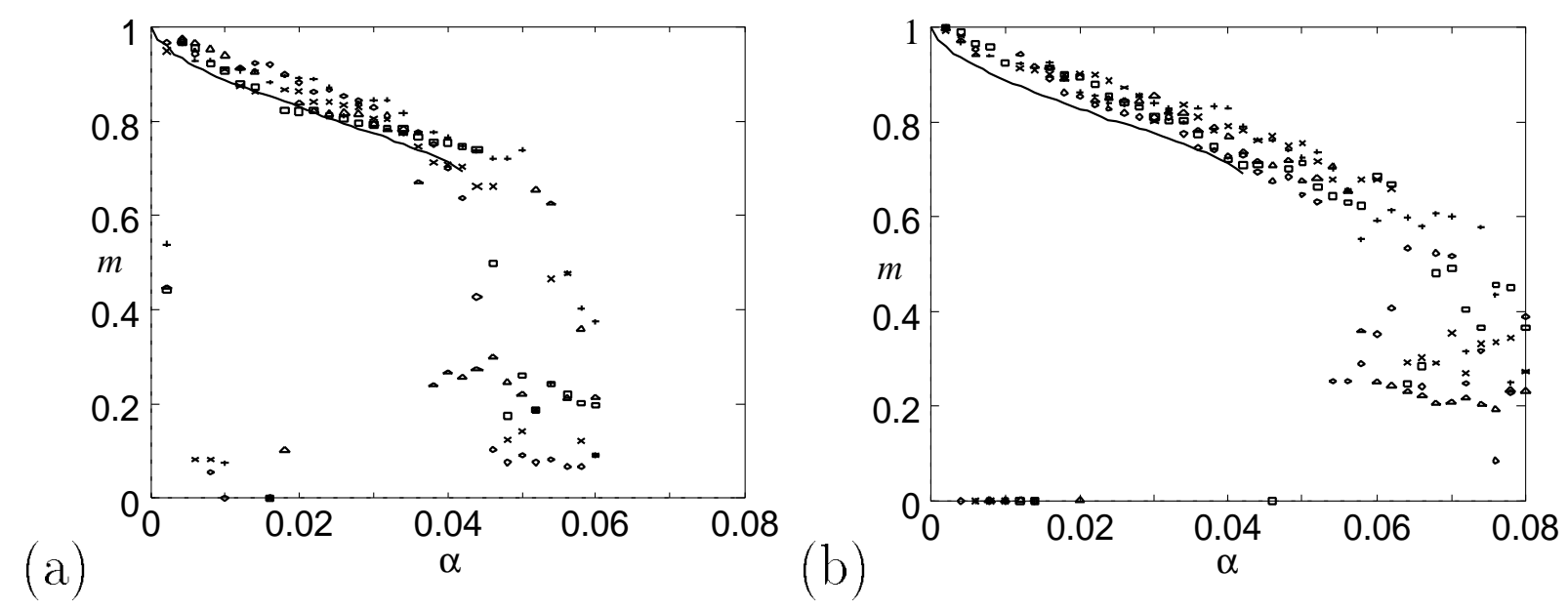

Figure 5

T. Aonishi 

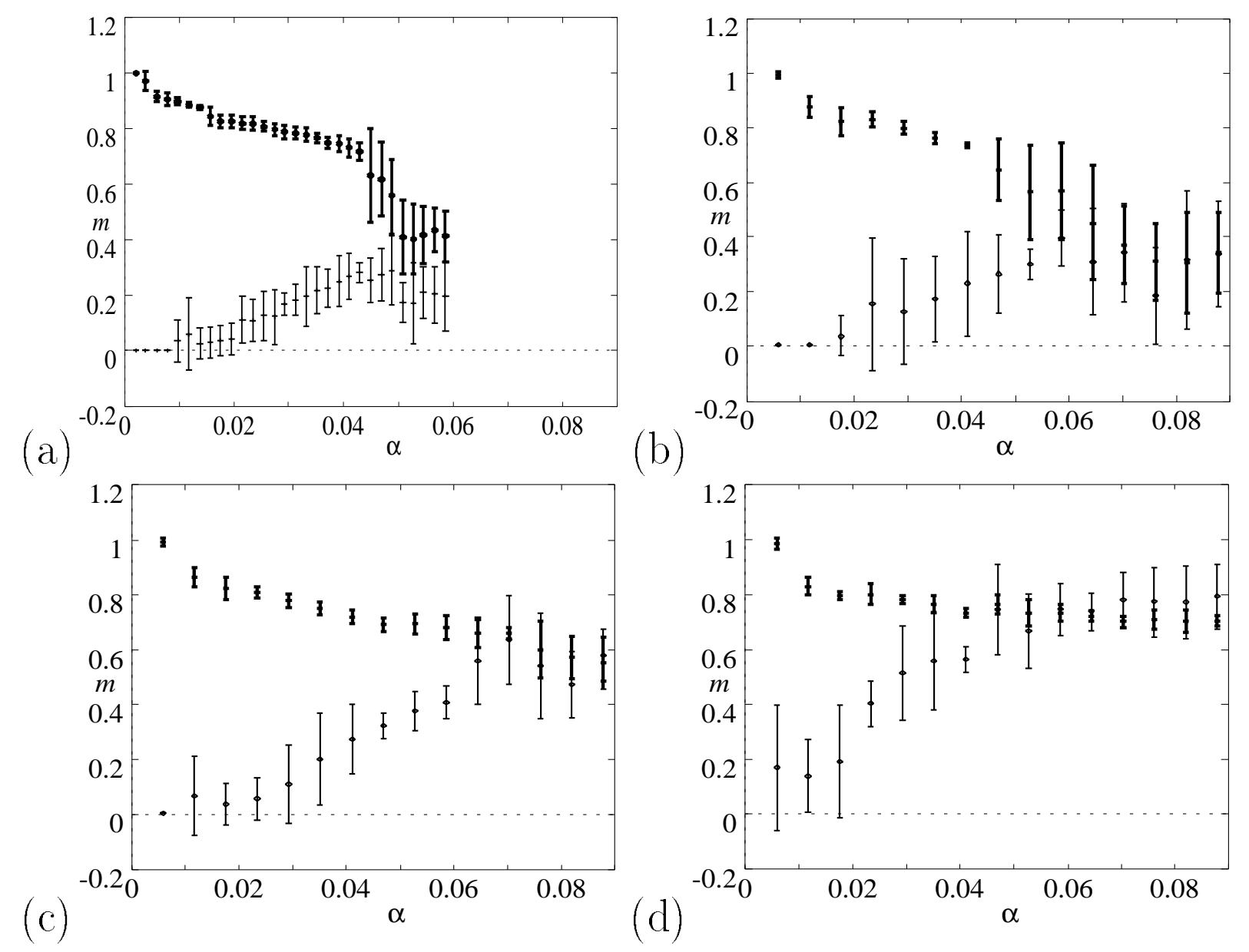

Figure 6

T. Aonishi 